UVX 2008 (2009) 139-144

(C) EDP Sciences, 2009

DOI: $10.1051 /$ uvx/2009022

\title{
Émission cohérente de sillage et dynamique plasma
}

\author{
C. Thaury ${ }^{1}$, F. Quéré ${ }^{1}$, H. George ${ }^{1}$, R.A. Loch², J.-P. Geindre ${ }^{3}$, G. Bonnaud ${ }^{1}$, \\ P. Monot ${ }^{1}$ et Ph. Martin ${ }^{1}$ \\ ${ }^{1}$ Service des Photons, Atomes et Molécules, IRAMISIDSM, CEA Saclay, \\ 91191 Gif-sur-Yvette Cedex, France \\ ${ }^{2}$ Laser Physics and Nonlinear Optics Group, Faculty of Science and Technology, \\ MESA+ Institute for Nanotechnology, University of Twente, The Netherlands \\ ${ }^{3}$ Laboratoire pour l'Utilisation des Lasers Intenses, CNRS, École Polytechnique, \\ 91128 Palaiseau, France
}

\begin{abstract}
Résumé. L'étude des propriétés des harmoniques d'ordres élevées générées sur miroir plasma peut fournir de nombreuses informations sur la dynamique du milieu d'interaction. Nous présentons dans cette article le principe de l'émission cohérente de sillage qui est le mécanisme de génération d'harmoniques sur miroir plasma le plus efficace en régime non relativiste. Nous utilisons ensuite une méthode interférométrique pour mesurer dans ce régime, la variation de la phase harmonique avec l'éclairement laser. Des simulations particulaires nous permettent enfin de relier cette variation à la dynamique électronique du plasma.
\end{abstract}

\section{INTRODUCTION}

Lorsqu' un faisceau laser intense est focalisé sur une cible solide, cette dernière est rapidement ionisée et un plasma se forme à sa surface. Ce plasma se comporte alors comme un miroir pour le laser. Pour des intensités supérieures à quelques $10^{15} \mathrm{~W} \mathrm{~cm}^{-2}$ et un contraste temporel suffisamment élevé [1], le spectre du faisceau réfléchi ne contient pas seulement la fréquence du laser incident, mais également des harmoniques d'ordres élevés de cette fréquence, associées dans le domaine temporel à un train d'impulsions attosecondes $\left(1\right.$ as $\left.=10^{-18} s\right)$.

Les simulations numériques montrent en effet, que le «miroir plasma » formé durant l'interaction, rayonne une impulsion attoseconde par période laser. Les propriétés de cette impulsion sont largement déterminées par la dynamique du plasma à l'instant de l'émission. En comparant les caractéristiques des différentes impulsions attosecondes formant le train, ou de façon équivalente les propriétés de phase des harmoniques individuelles, on peut donc obtenir des informations sur la dynamique électronique du plasma.

Dans cet article, nous montrons tout d'abord qu'en régime non relativiste, le modèle de l'émission cohérente de sillage permet de relier l'émission d'une impulsion attoseconde, à la traversée du plasma par des électrons énergétiques. La mesure de la phase des harmoniques individuelles donne donc accès à la variation au cours de l'impulsion laser, du temps de retour de ces électrons. Nous illustrons ensuite comment cette phase peut être évaluée précisément par une méthode interférométrique et nous utilisons cette mesure pour suivre la dynamique électronique du plasma avec une précision de quelques dizaines d'attosecondes.

\section{2. ÉMISSION COHÉRENTE DE SILLAGE}

En régime non relativiste les harmoniques générées lors de l'interaction d'un laser femtoseconde avec un miroir plasma sont essentiellement produites par émission cohérente de sillage (Coherent Wake Emission) [2,3]. Ce mécanisme peut se décomposer en trois étapes, qui sont illustrées par les figures 1 et 2. Ces figures sont issues d'une simulation particulaire réalisée avec le code 1D3V EUTERPE [4], lors de laquelle une impulsion laser polarisée $p$ avec un potentiel vecteur $a_{0}=0.2\left(I=5 \times 10^{17} \mathrm{~W} \mathrm{~cm}^{-2}\right.$ à 

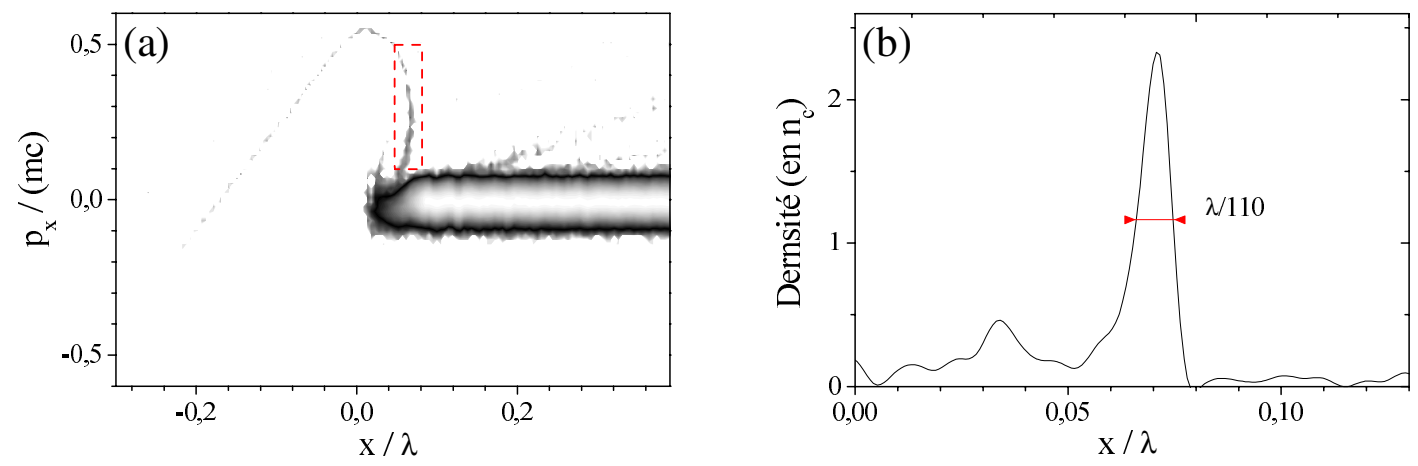

Figure 1. Pic de densité électronique dans l'espace des phases. (a) Espace des phases $\left(x, p_{x}\right)$. On observe une concentration d'électron en $x \approx 0.07 \lambda$ pour $p_{x} \gtrsim 0.1 \mathrm{mc}$. (b) Somme sur $p_{x}>0.1 \mathrm{mc}$ de la densité électronique de l'espace des phases en (a). Les électrons énergétiques sont concentrés sur une distance inférieure à un centième de la longueur d'onde laser.

$800 \mathrm{~nm}$ ), est envoyée sur un plasma de densité électronique maximale $n_{e}^{\max }=225 n_{c}$, où $n_{c}$ est la densité critique pour la fréquence laser. Les ions sont gardés immobiles et un gradient exponentiel de $0.017 \lambda$, où $\lambda$ est la longueur d'onde laser, est imposé à la surface du plasma. Enfin, un angle d'incidence de $45^{\circ}$ est simulé en utilisant la méthode de Bourdier [5].

On observe sur la figure 1(a), une carte de l'espace des phases électronique $\left(x, p_{x}\right)$, où $O x$ est l'axe normal au plasma et $p_{x}$ la projection de l'impulsion sur cet axe. Cette carte a été construite à un temps du cycle optique laser, où les électrons de Brunel, qui ont été tirés dans le vide par le champ électrique, sont renvoyés vers le plasma et traversent le gradient de densité à sa surface [6]. On remarque qu'une partie importante de ces électrons rapides est concentrée sur une petite distance $\Delta x_{p}$. Ceci est illustré par la figure 1(b), où est tracé le profil de densité des électrons énergétiques intégré sur l'intervalle $p_{x}>0.1 \mathrm{mc}$. Cette figure met en effet en évidence l'existence d'un pic de densité électronique, dense et ultracourt, s'étalant sur moins d'un centième de la longueur d'onde laser. Ce pic de densité est formé par des croisements de trajectoires d'électrons de Brunel, qui se produisent lorsque les électrons les plus rapides rattrapent ceux qui sont retournés vers le plasma avant eux (voir la figure 1(a)). Dans les conditions de cette simulation, ce pic se propage à une vitesse $v_{p} \approx 0.25 c$ sur une distance de l'ordre de $\lambda / 5$, au delà de laquelle les électrons de Brunel ne se croisent plus.

Les trajectoires des électrons qui se croisent en $x=0.06 \lambda$ sont représentées sur la figure 2, superposées à une carte de champ $E_{x}$. Cette carte de champ met en évidence la présence d'oscillations plasmas dans le gradient de densité $(x>0)$, qui ont été excitées dans le sillage du pic de densité électronique. La fréquence de ces oscillations plasmas $\omega_{p}(x)=\left(n_{e}(x) e^{2} / m \epsilon_{0}\right)^{1 / 2}$ augmente au fur et à mesure que l'on s'enfonce dans le gradient, jusqu'à atteindre sa valeur maximale $\omega_{p}^{\max }=$ $\left(n_{e}^{\max } / n_{c}\right)^{1 / 2} \omega_{0}=15 \omega_{0}$, où $\omega_{0}$ est la pulsation laser. On peut noter que cette excitation est efficace sur toute la longueur du gradient en raison de la durée extrêmement courte des pics : $\Delta x_{p} / v_{p} \approx T / 30<$ $2 \pi / \omega_{p}^{\max }$, où $T$ est la période du laser.

Parce qu'elles sont excitées dans un plasma non-homogène, ces oscillations plasmas engendrent des courants transverses qui peuvent rayonner des ondes électromagnétiques. Cette émission se fait par conversion linéaire de mode [7], les oscillations plasmas en $x$ se couplant avec une onde électromagnétique de pulsation $\omega_{p}(x)$. En raison des conditions d'accord de phase, ce processus n'est efficace qu'au voisinage du temps où les fronts d'onde des oscillations plasmas admettent une tangente horizontale [2]. Ainsi, l'émission se fait de façon transitoire, sous la forme d'impulsions attosecondes (voir la figure 2). Une impulsion attoseconde est émise durant chaque cycle optique, peu après le passage d'un pic de densité électronique. On génère donc un train d'impulsions séparées d'une période laser, associé dans le domaine spectral à un spectre d'harmoniques de la fréquence du laser. 


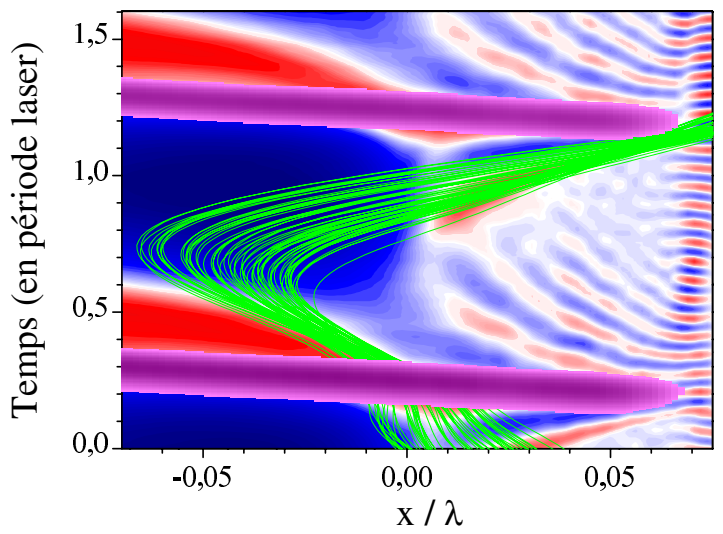

Figure 2. Excitation d'oscillations plasmas par des pics de densité, formés par des électrons de Brunel. On observe les trajectoires des électrons énergétiques qui forment un pic de densité dans le gradient, tracées sur une carte de champ $E_{x}$. On voit que ces électrons excitent des oscillations plasmas dans le gradient de densité. Les oscillations entre $t \approx 0$ et $t \approx 1 T$ ont été excitées par les électrons de Brunel revenus lors du cycle laser précédent. Ces oscillations plasmas émettent un train d'impulsions attosecondes, avec une nouvelle impulsion émise peu après le passage de chaque pic de densité (échelle de couleur pourpre).

Cette analyse montre que l'instant d'émission d'une impulsion attoseconde est déterminé par le temps de passage des électrons de Brunel dans le gradient de densité. Une variation de ce temps, durant la durée de l'impulsion laser, entraîne une perte de périodicité qui se traduit dans le domaine spectral par une phase harmonique non-linéaire [8]. Nous montrerons dans la section 4 que cette phase peut se mesurer par une méthode interférométrique, donnant ainsi accès à la variation du temps de retour dans le plasma des électrons de Brunel.

\section{COHÉRENCE MUTUELLE}

Toute expérience d'interférométrie suppose que l'on est capable de produire plusieurs sources mutuellement cohérentes. Cette opération est généralement effectuée par division de front d'onde ou d'amplitude. Dans cette section, nous présentons une méthode originale, consistant à former dans le plan focal laser trois taches verrouillées en phase, ce qui permet d'obtenir trois sources harmoniques mutuellement cohérentes.

Le schéma du montage utilisé est représenté sur la figure 3(a). Des impulsions laser de $60 \mathrm{fs}$, ayant un très haut contraste temporel grâce à un double miroir plasma [9], sont focalisées à l'aide d'un miroir parabolique sur une cible de silice, pour atteindre un éclairement allant de quelques $10^{16} \mathrm{Wcm}^{-2}$ à quelques $10^{17} \mathrm{~W} \mathrm{~cm}^{-2}$. Le faisceau réfléchi est ensuite filtré spectralement pour sélectionner un groupe d'harmoniques, puis envoyé sur des galettes de microcanaux, elles mêmes imagées par une caméra CCD (Charge-Coupled Device) qui fourni une image 2D du faisceau harmonique. Dans le trajet du faisceau incident, on a placé un diaphragme en forme de peigne qui est conçu de façon à obtenir au foyer, trois taches focales synchronisées en phase, séparées de $a=40 \mu m$ (voir la figure 3(b)). Les deux taches latérales sont identiques à la tache centrale, à leur amplitude près.

Ces trois taches forment trois sources d'harmoniques qui interfèrent sur le détecteur situé à la distance $D=38 \mathrm{~cm}$ de la cible. On observe ainsi sur le profil spatial du faisceau harmonique des franges d'interférences (figures 3(c-d)). Leur contraste est excellent $(\approx 80 \%)$, ce qui prouve que l'on peut générer plusieurs sources mutuellement cohérentes, sur miroir plasma. Nous exploitons maintenant cette propriété pour mesurer la variation avec l'éclairement laser de la phase des harmoniques individuelles. 

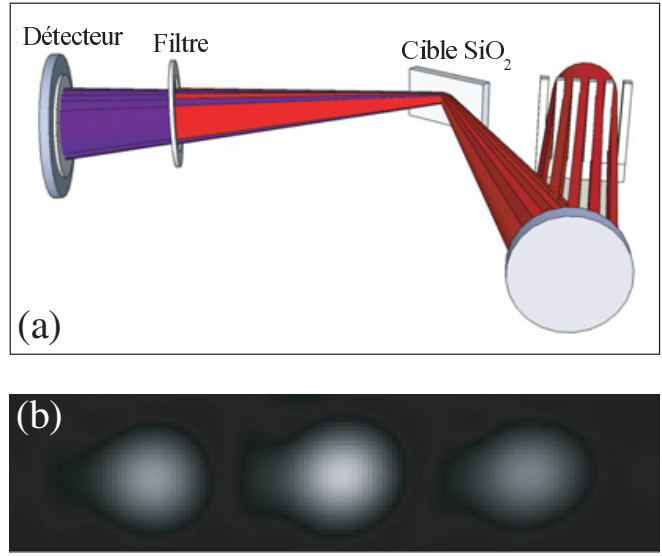
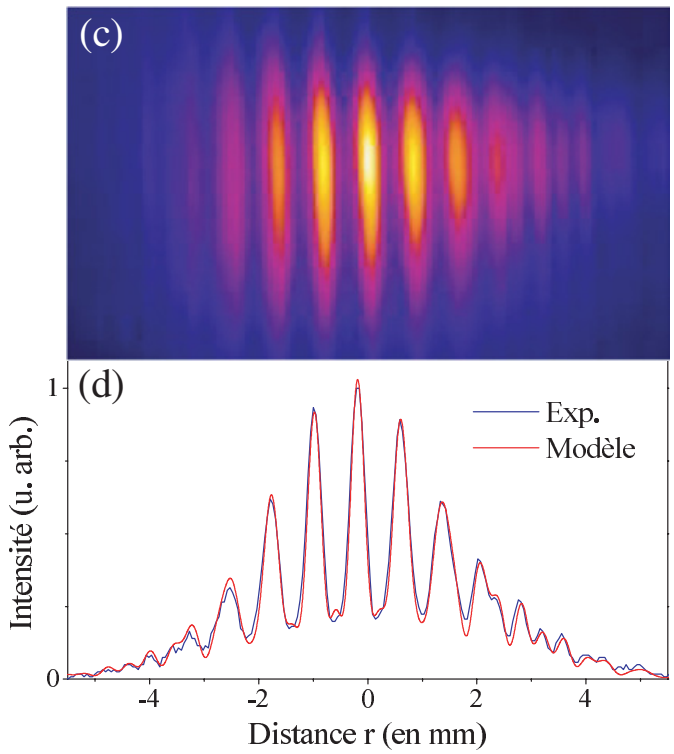

Figure 3. Interférences à trois sources harmoniques. (a) Schéma expérimental simplifié. Des lames de silice traitées anti-reflet permettant d'éliminer en parti le faisceau infrarouge réfléchi ont été omises. (b) Image de la distribution d'intensité au foyer. (c) Figures d'interférences obtenues au niveau des galettes à $D=38 \mathrm{~cm}$ de la source pour une superposition des harmoniques 8 à 10 (filtre d'indium). Le faisceau laser est ouvert à $f / 17$. (d) Profils des franges. La courbe expérimentale a été extraite de (c), la courbe théorique a été obtenue pour $\Delta \phi_{n \omega_{0}}=0.23 \times n \mathrm{rad}$.

\section{DYNAMIQUE ÉLECTRONIQUE DU PLASMA}

Les interférences entre trois sources de différentes intensités, peuvent être décrites par l'équation suivante [10]:

$$
I(\mathbf{r})=\int d \omega F_{\omega}(\mathbf{r})\left[1+2 \alpha_{\omega}+4 \sqrt{\alpha_{\omega}} \cos \left(\Delta \phi_{\omega}\right) \cos \left(\mathbf{k}_{\omega} \mathbf{r}\right)+2 \alpha_{\omega} \cos \left(2 \mathbf{k}_{\omega} \mathbf{r}\right)\right]
$$

où $\mathbf{k}_{\omega}=(\omega / c D) \mathbf{a}$ et où $F_{\omega}(\mathbf{r})$ est le profil spatial en intensité à la fréquence $\omega$, obtenu dans le plan de détection dans le cas d'une source unique. $\alpha_{\omega}$ est le rapport d'éclairement entre la source centrale et ses satellites, et $\Delta \phi_{\omega}=\phi_{\omega}(I)-\phi_{\omega}\left(\alpha_{\omega_{0}} I\right)$ le déphasage entre ces sources. Cette équation montre que pour chaque pulsation $\omega$, la figure d'interférence contient deux jeux d'interférences, de fréquences spatiales $\left|\mathbf{k}_{\omega}\right|$ et $2\left|\mathbf{k}_{\omega}\right|$. Les franges de fréquence $\left|\mathbf{k}_{\omega}\right|$ sont associées aux interférences de la source harmonique centrale avec les deux latérales, alors que celles de fréquence $2\left|\mathbf{k}_{\omega}\right|$ correspondent aux interférences entre les deux satellites. Dans le cas plus usuel des interférences à deux sources, un déphasage se traduit par un décalage spatial des franges. Dans le cas considéré ici, la symétrie de la configuration fait que le terme de phase $\Delta \phi_{\omega}$ affecte le contraste des franges de fréquence $\left|\mathbf{k}_{\omega}\right|$, mais pas leurs positions.

Ce déphasage peut être extrait des interférogrammes, en effectuant une analyse de Fourier [10]. La figure 4(a) montre le résultat de cette mesure, pour les harmoniques 11 à 13 , et différents rapports d'éclairements $\alpha_{\omega_{0}}$. On observe que $\Delta \phi_{\omega}$ diminue de $\approx \pi$ jusqu'à pratiquement $\pi / 2$, lorsque $\alpha_{\omega_{0}}$ varie de 0.57 à 0.74 . On voit par ailleurs que le déphasage $\Delta \phi_{\omega}$ entre la source harmonique centrale et les deux latérales, mesuré pour chaque $\alpha_{\omega_{0}}$, varie linéairement avec la fréquence harmonique. Ceci est caractéristique, dans le domaine temporel, d'un délai $\tau=\Delta \phi_{\omega} / \omega$. Ainsi cette expérience prouve que les champs $E^{0}(t)$ et $E^{1}(t)$, associés aux trains d'impulsions attosecondes, émis respectivement par la tache centrale et les taches latérales, sont identiques à leur amplitude près, et simplement décalés en temps de $\tau: E^{0}(t) \propto E^{1}(t+\tau)$. Les variations de ce délai avec le rapport d'éclairement sont représentées sur 

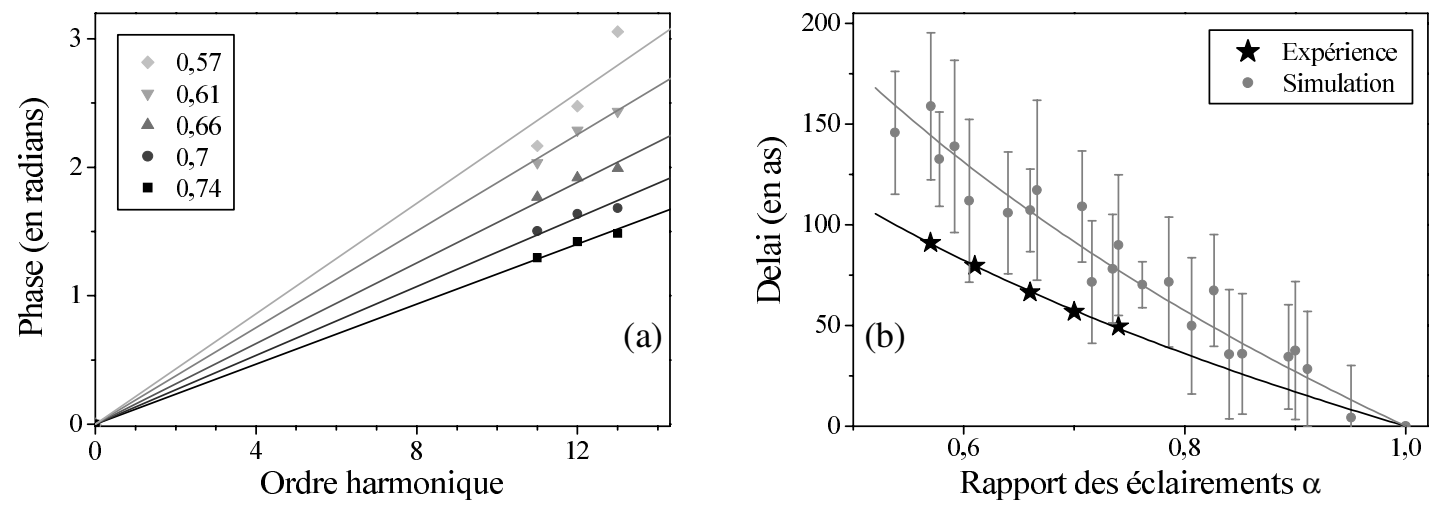

Figure 4. Mesure expérimentale de la phase harmonique. (a) Variation du déphasage $\Delta \phi_{\omega}$ avec l'ordre harmonique, pour les 5 valeurs de $\alpha_{\omega_{0}}$ indiquées en légende. Chaque point correspond à une moyenne sur 5 tirs. L'incertitude est d'environ 10\%. (b) Délai entre les impulsions attosecondes générées sur la tache centrale et les taches latérales, en fonction de $\alpha_{\omega_{0}}$. Ces résultats sont comparés avec les délais calculés à partir de simulations particulaires effectuées pour des conditions d'éclairements comparables. Dans ce cas, les barres d'erreurs sont dues aux fluctuations du délai le long du train d'impulsions attosecondes.

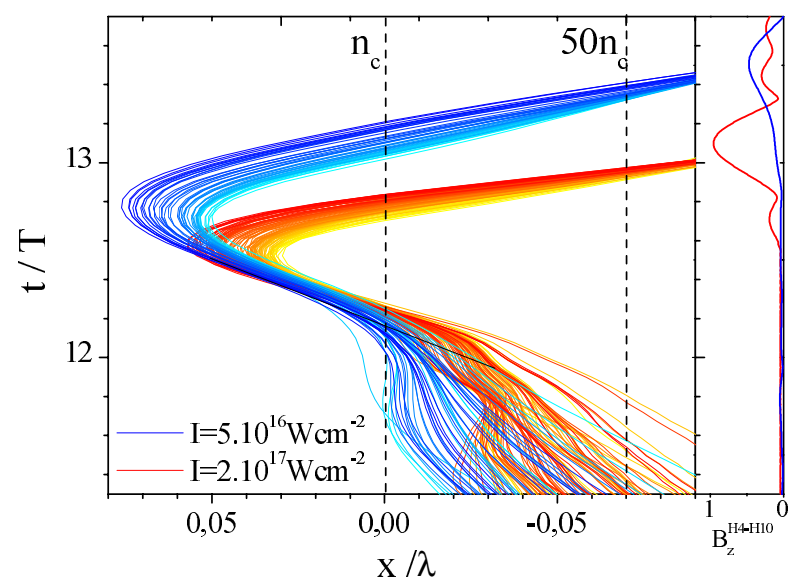

Figure 5. Variation avec l'éclairement laser du temps de retour dans le plasma des électrons de Brunel. Les trajectoires $(x, t)$ des électrons qui forment un pic de densité dans le gradient ont été tracées pour deux éclairements différents. Dans les deux cas les électrons ont été pris au hasard, parmi ceux qui se trouvent dans la boite tracée en traits pointillés sur l'espace des phases de la figure 1, au moment où le pic de densité traverse le gradient. Sur le panneau de droite, on observe les deux impulsions attosecondes émises suite au passage de ces pics de densité.

la figure 4(b). On observe qu'il diminue lorsque $\alpha_{\omega_{0}}$ se rapproche de 1, tendant vers 0 lorsque les trois sources sont identiques.

Les simulations particulaires permettent d'expliquer ce délai. La figure 5 montre effectivement que les électrons qui se croisent dans le gradient de densité, mettent plus de temps à revenir vers le plasma à faible éclairement laser. Ainsi, les oscillations plasmas sont excitées plus tard, et par suite les impulsions attosecondes émises plus tard lorsque l'éclairement laser diminue. D'après les simulations, ce phénomène est la principale conséquence d'une variation de l'éclairement laser, sur le train d'impulsions attosecondes [8]. Ceci est en accord avec les résultats expérimentaux précédents qui montrent que seul le temps d'émission des impulsions attosecondes varie avec l'éclairement. 
Les délais mesurés expérimentalement sont comparés sur la figure 4(b), avec des points théoriques issus de simulations particulaires EUTERPE. Les valeurs expérimentales et numériques diffèrent d'un facteur $\approx 1.6$, mais l'évolution de $\tau$ avec $\alpha_{\omega_{0}}$ est similaire dans les deux cas. Cet accord qualitatif est satisfaisant, puisque qu'un code particulaire est simplement un modèle de l'interaction laser-plasma, qui ne prend pas en compte tous les processus physiques. Ce bon accord global, prouve qu'il est possible de mesurer les variations du temps de retour des électrons de Brunel avec l'éclairement laser, avec une précision de l'ordre de quelques dizaines d'attosecondes.

Ces résultats peuvent être également exploités pour mesurer les variations de la phase harmonique avec l'éclairement laser, et ainsi évaluer la dérive de fréquence des harmoniques. En effet, les simulations numériques montrent que le déphasage peut se mettre sous la forme $\Delta \phi_{n \omega_{0}}=A_{n} \ln \left(\alpha_{\omega_{0}}\right)$. Ainsi, en utilisant les données expérimentales pour estimer $A_{n}$ (voir les courbes de tendance de la figure 4), on peut modéliser les variations de la phase harmonique avec l'éclairement laser. En utilisant les points expérimentaux, on trouve que $A_{n} \approx-0.37 n$, ce qui permet d'estimer, en supposant que l'émission dure environ $60 \mathrm{fs}$, que le paramètre de dérive de fréquence $d^{2} \phi_{n \omega_{0}} / d t^{2} \approx-2 n \times$ $10^{-4} \mathrm{PHz} \mathrm{fs}^{-1}$. Cette valeur négative correspond à une dérive de fréquence du bleu vers le rouge. On peut noter que ce résultat est en accord qualitatif avec l'évaluation effectuée dans la référence [2], dans des conditions expérimentales légèrement différentes $\left(d^{2} \phi_{n \omega_{0}} / d t^{2} \approx-8 n \times 10^{-4} \mathrm{PHz} \mathrm{fs}^{-1}\right)$.

En conclusion, nous avons montré que la génération d'harmoniques sur miroir plasma peut être utilisée pour sonder la dynamique d'un plasma interagissant avec une impulsion laser intense. Nous avons illustré le potentiel de cette approche en mesurant, dans le cas de l'émission cohérente de sillage, la dépendance en éclairement de la phase des harmoniques individuelles. De nombreuses autres informations sur les différentes étapes de l'interaction pourraient être obtenues en caractérisant complètement le champ rayonné par le plasma, avec une précision attoseconde.

\section{Références}

[1] P. Monot, G. Doumy, S. Dobosz, M. Perdrix, P. D’Oliveira, F. Quéré, F. Réau, Ph. Martin, P. Audebert, J. C. Gauthier, and J. P. Geindre. High-order harmonic generation by nonlinear reflection of an intense high-contrast laser pulse on a plasma. Optics Letter, 29:893, 2004.

[2] F. Quéré, C. Thaury, P. Monot, S. Dobosz, Ph. Martin, J.-P. Geindre, and P. Audebert. Coherent Wake Emission of High-Order Harmonics from Overdense Plasmas. Physical Review Letters, 96(12):125004, March 2006.

[3] C. Thaury, F. Quéré, J.-P. Geindre, A. Levy, T. Ceccotti, P. Monot, M. Bougeard, F. Réau, P. D'Oliveira, P. Audebert, R. Marjoribanks, and Ph. Martin. Plasma mirrors for ultrahighintensity optics. Nature Physics, 3:424-429, June 2007.

[4] G. Bonnaud and G. Reisse. Particle code study of the influence of non-monochromaticity of laserlight on stimulated raman-scattering in laser-irradiated plasmas. Nuclear Fusion, 5(26):633-646, 1986.

[5] A. Bourdier. Oblique incidence of a strong electromagnetic wave on a cold inhomogeneous electron plasma - Relativistic effects. Physics of Fluids, 26:1804-1807, July 1983.

[6] F. Brunel. Not-so-resonant, resonant absorption. Physical Review Letter, 59(1):52-55, Jul 1987.

[7] D. E. Hinkel-Lipsker, B. D. Fried, and G. J. Morales. Analytic expressions for mode conversion in a plasma with a linear density profile. Physics of Fluids B, 4:559-575, March 1992.

[8] F. Quéré, C. Thaury, J.-P. Geindre, G. Bonnaud, P. Monot, and Ph. Martin. Phase Properties of Laser High-Order Harmonics Generated on Plasma Mirrors. Physical Review Letters, 100(9):095004-+, March 2008.

[9] A. Lévy, T. Ceccotti, P. D’Oliveira, F. Réau, M. Perdrix, F. Quéré, P. Monot, M. Bougeard, H. Lagadec, Ph. Martin, P. Audebert, and J.P. Geindre. Double plasma mirror for ultrahigh temporal contrast ultraintense. Optics Letter, 32(3):310, February 2007.

[10] C. Thaury, H. George, F. Quéré, R. Loch, J.-P. Geindre, P. Monot, and Ph. Martin. Coherent dynamics of plasma mirrors. Nature Physics, 4, August 2008. 